\title{
Fractal features of oscillatory convection in the half-floating zone
}

\author{
ZE-MEI TANG and WEN-RUI HU \\ Institute of Mechanics, Chinese Academy of Sciences, 15 Zhong Guan Cun Road, Beijing 100080 , \\ People's Republic of China
}

(Received 12 September 1994)

\begin{abstract}
Unsteady and two-dimensional numerical simulation is applied to study the transition process from steady convection to turbulence via subharmonic bifurcation in thermocapillary convection of a liquid bridge in the half-floating zone. The results of numerical tests show clearly the fractal structure of period-doubling bifurcations, and frequency-locking at $f / 4, f / 8, f / 16$ with basic frequency $f$ is observed with increasing temperature difference. The Feigenbaum universal constant is given by the present paper as $\delta_{4}=4.853$, which can be compared with the theoretical value 4.6642016 .
\end{abstract}

\section{INTRODUCTION}

The route to turbulent convection is a typical subject in the fluid mechanics of a dissipative system. The Rayleigh-Benard convection induced by the buoyancy has been studied extensively (see, for example, in refs [1-3]), although open problems of the subject are still attractive at the present time. There are many ways to determine the turbulence of the convection, depending on the typical parameters such as the Rayleigh number, the Prandtl number and geometric aspect. From the viewpoint of theory, the transition process of a dissiparive system other than the Benard convection will be interesting.

Thermocapillary convection driven by the non-uniformity of surface tension on the free surface is another typical dissipative system, and has been studied particularly in microgravity fluid mechanics. Groundbased studies of thermocapillary convection in the liquid bridge of a half-floating zone, open-liquid disk or multi-layers of liquid medium were carried out to emphasize the thermocapillary effect in comparison with the buoyancy effect by reducing the typical scale. Half-floating-zone convection is a subject of great interest from the viewpoint of both the dissipative system on theoretical side and the space materials processes on the application side. The studies of the transition process from steady state to oscillatory state for convection in the half-floating zone have concentrated on the onset of oscillation. The experiments were completed mostly by measurement of temperature, using an inserted thermocouple inside the liquid bridge or near the free surface [4-6]. Recently, measurements of free surface waves were also presented $[7,8]$. All experiments gave the features near the onset of oscillation, which is far from a complete turbulence. The instability analyses suggested the mechanism of hydrothermal wave instability [9] and surface wave instability [10] for a simplified onedimensional basic state. The analyses extended to the two-dimensional basic state with symmetric assumption [11] and for non-symmetric cases [12]. The influence of the gravitational effect on the onset of oscillation, even for the case with small Bond numbers, was also analyzed, and the results showed the important effects of gravity [13]. On the other hand, some studies suggested that the oscillatory convection in the halffloating zone is not induced by instability, but comes from the different periods mass and heat flux [14]. Therefore, the subject of onset oscillation, which is only the initial period of transition to turbulence, is still an open problem.

In the present paper, a complete route to turbulent convection is presented by the method of numerical simulation for two-dimensional and unsteady mode of the half-floating zone. Numerical test is concentrated on the bifurcation feature during the transition process. Subharmonic bifurcation is obtained by calculation, and the Feigenbaum universal constant is given close to the theoretical value. The physical model and numerical simulation are discussed in the next section, and the bifurcation feature is presented in Section 3. The last section contains conclusions and discussions.

\section{PHYSICAL MODEL AND MATHEMATICAL DESCRIPTION}

The two-dimensional half floating zone consists of a liquid column floating in a gap between two parallel planes with the same width, $D_{0}=4 \mathrm{~mm}$, and infinite extension. The upper plane has relatively high temperature $T_{2}^{\prime}$ in comparison with the lower one $T_{1}^{\prime}$. The geometric aspect is adopted as $L / D_{0}=1$, with the minimum width of the liquid bridge $D_{\min }=0.9 D_{0}$, where $L$ is the height of liquid bridge, as shown in 


\section{NOMENCLATURE}

$A \quad$ geometrical aspect

$B_{0} \quad$ static Bond number

$D_{\min } \quad$ minimum width of liquid bridge

$D_{0} \quad$ width of liquid bridge at upper or lower wall

$f \quad$ basic frequency of oscillation

$g$ gravitational acceleration

$G_{\mathrm{r}} \quad$ Grashof number

$H$ function of free surface shape

$k$ thermal diffusion coefficient

$L$ height of liquid bridge

$M_{\text {a }} \quad$ Marangoni number

$P_{\mathrm{r}} \quad$ Prandtl number

$R_{\mathrm{s}} \quad$ Reynolds number

$t$ dimensionless time

$t^{\prime} \quad$ dimensional time

$T$ dimensionless temperature

$T^{\prime} \quad$ dimensional temperature

$T_{1}^{\prime} \quad$ dimensional temperature of lower wall

$T_{2}^{\prime} \quad$ dimensional temperature of upper wall

$u$ horizontal component of dimensionless velocity $u^{\prime} \quad$ horizontal component of dimensional velocity

$U_{\mathrm{r}}^{\prime} \quad$ reference velocity

$v \quad$ axial component of dimensionless velocity

$v^{\prime} \quad$ axial component of dimensional velocity

$x \quad$ dimensionless abscissa coordinate

$x^{\prime} \quad$ dimensional abscissa coordinate

$y \quad$ dimensionless axial coordinate

$y^{\prime}$ dimensional axial coordinate.

Greek symbols

$\beta \quad$ thermal expansion coefficient

$\Delta T^{\prime} \quad$ applied temperature difference

$\Delta T_{\mathrm{c}}^{\prime} \quad$ critical applied temperature difference

$v \quad$ viscosity coefficient

$\rho$ density of fluid

$\sigma \quad$ surface tension coefficient

$\psi \quad$ stream-function

$\omega$ vorticity.
Fig. 1. The direction of the gravity vector is opposite to the $y$-axis. The configuration of the liquid bridge is determined by the static equilibrium condition and assumed to be unchanged in the computational process. The Boussinesq approximation for an incompressible fluid is used. The Navier-Stokes equation and the energy equation described by dimensionless vorticity $\omega$, stream function $\psi$ and temperature $T$ are applied for the convection and heat transfer processes in the liquid bridge. The height of the liquid bridge $L$ is adopted as the characteristic length. The typical velocity $U_{\mathrm{r}}^{\prime}=\left|\partial \sigma / \partial T^{\prime}\right|\left(T_{2}^{\prime}-T_{1}^{\prime}\right) / \rho \nu$ is introduced from the balance of the tangential shear stresses at the free surface, where $T_{2}^{\prime}-T_{1}^{\prime}$ is the applied temperature

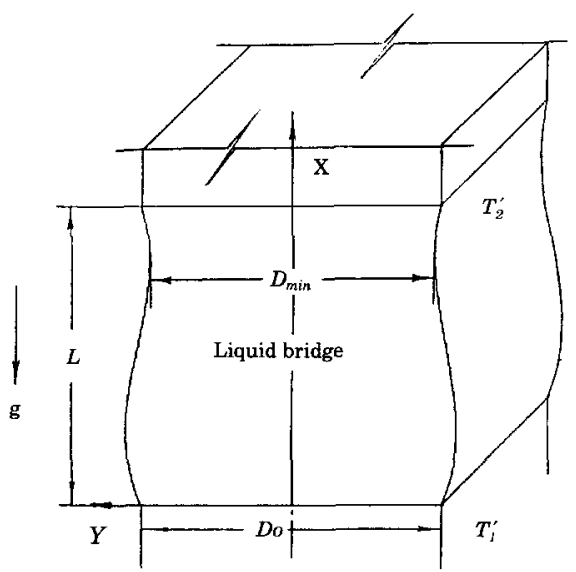

Fig. 1. Schematic diagram of a two-dimensional liquid bridge. difference between the top and the bottom walls. The dimensionless quantities may be defined as follows.

$$
\begin{gathered}
x=\frac{x^{\prime}}{L} \quad y=\frac{y^{\prime}}{L} \quad t=\frac{t^{\prime} U_{\mathrm{r}}^{\prime}}{L} \quad U_{\mathrm{r}}^{\prime}=\frac{\left|\frac{\partial \sigma}{\partial T^{\prime}}\right|\left(T_{2}^{\prime}-T_{1}^{\prime}\right)}{\rho v} \\
u=\frac{u^{\prime}}{U_{\mathrm{r}}^{\prime}} \quad v=\frac{v^{\prime}}{U_{\mathrm{r}}^{\prime}} \quad T=\frac{T^{\prime}}{\left(T_{2}^{\prime}-T_{1}^{\prime}\right)} \quad B_{0}=\frac{\boldsymbol{g} \rho L^{2}}{\sigma} \\
R_{\mathrm{s}}=\frac{U_{\mathrm{r}}^{\prime} L}{v} \quad M_{\mathrm{a}}=\frac{U_{\mathrm{r}}^{\prime} L}{\kappa} \quad G_{\mathrm{r}}=\frac{\boldsymbol{g} \beta\left(T_{2}^{\prime}-T_{1}^{\prime}\right) L^{3}}{v^{2}}
\end{gathered}
$$

where superscript prime expresses dimensional quantities, for example, $\left(u^{\prime}, v^{\prime}, 0\right)$ is the dimensional velocity vector, $\sigma, \beta, v, \kappa, \partial \sigma / \partial T$, and $g$ are, respectively, the surface tension coefficient, thermal expansion coefficient, kinematic viscosity, thermal diffusion coefficient, differentiation of surface-tension respected to temperature, and the Earth's gravitational acceleration. The non-dimensional parameters involve static Bond number $B_{0}$, the Grashof number $G_{\mathrm{r}}$, the Reynolds number $R_{\mathrm{s}}$ based on the typical velocity $U_{\mathrm{r}}^{\prime}$, and Prandtl number $P_{\mathrm{r}}=v / \kappa$. The Marangoni number, Reynolds number and Prandtl number are connected by $M_{\mathrm{a}}=R_{\mathrm{s}} \cdot P_{\mathrm{r}}$.

The stream function $\psi$ and vorticity $\omega$ are introduced, respectively, in non-dimensional Cartesian coordinates $(x, y, z)$ as follows:

$$
u=\frac{\partial \psi}{\partial y} \quad v=-\frac{\partial \psi}{\partial x} \quad w=0
$$




$$
\omega=\frac{\partial v}{\partial x}-\frac{\partial u}{\partial y}
$$

Then, the dimensicnless equations for $\psi, \omega$ and temperature $T$ may be written as [15]:

$$
\begin{gathered}
\frac{\partial^{2} \psi}{\partial x^{2}}+\frac{\partial^{2} \psi}{\partial y^{2}}=-\omega \\
R_{\mathrm{s}}\left(\frac{\partial \omega}{\partial t}+\frac{\partial \psi}{\partial y} \frac{\partial \omega}{\partial x}-\frac{\partial \psi}{\partial x} \frac{\partial \omega}{\partial y}\right)=\frac{\partial^{2} \omega}{\partial x^{2}}+\frac{\partial^{2} \omega}{\partial y^{2}}-\frac{G_{\mathrm{r}}}{R_{\mathrm{s}}} \frac{\partial T}{\partial y} \\
M_{\mathrm{a}}\left(\frac{\partial T}{\partial t}+\frac{\partial \psi}{\partial y} \frac{\partial T}{\partial x}-\frac{\partial \psi}{\partial x} \frac{\partial T}{\partial y}\right)=\frac{\partial^{2} T}{\partial x^{2}}+\frac{\partial^{2} T}{\partial y^{2}} \\
\text { The boundary conditions may be simplified as follows } \\
\text { for the case of a fixed-free surface } \\
\psi(0, y, t)=\psi(1, y, t)=\psi[x, H(x), t]=0 \\
\omega(0, \mathrm{y}, \mathrm{t})=\omega(1, \mathrm{y}, \mathrm{t})=\frac{2\left(\psi_{k+1}-\psi_{k}\right)}{\Delta x^{2}} \\
\left.\omega\right|_{y=R(x)}=-\frac{4 H_{x} u_{x}}{\left(1-H_{x}^{2}\right)}+2 v_{x}+\frac{\left(1+H_{x}^{2}\right)}{\left(1-H_{x}^{2}\right)} \frac{\mathrm{d} T}{\mathrm{~d} S} \\
T(0, y, t)=0 \quad T(1, y, t)=1, \quad \frac{\partial T[x, H(x), t]}{\partial n}=0
\end{gathered}
$$

where the subscript $x$ denotes differentiation with respect to $x, \psi_{k+1}$ and $\psi_{k}$ are, respectively, the stream function values at the distance $\Delta x$ from the wall, and the equation of the free surface is described by

$$
y=H(x) \text {. }
$$

In equation (9), the vorticity at the free-surface is given by the equilibrium condition of tangential stresses. Then, the problem is reduced to solve equations (4)-(6) under boundary conditions (7)-(10) for certain parameter values.

The aim of the present calculations is to identify the route of transition. leading to turbulent convection from the steady thermocapillary convection in the half-floating zone. At first, the steady state $\left(\psi_{0}, \omega_{0}\right.$, $T_{0}$ ) corresponding to applied temperature difference $T_{2}^{\prime}-T_{1}^{\prime}=1^{\circ} \mathrm{C}$ are calculated as the initial values. The critical applied temperature difference $\left(T_{2}^{\prime}-T_{1}^{\prime}\right)_{c}$, which describes the onset from the symmetric and steady convection to periodic and asymmetric oscillatory convection, can be obtained during increased applied temperature difference, then a sequence of bifurcation may appear. The applied temperature difference is increased usually in a step $\delta T^{\prime}=0.5^{\circ} \mathrm{C}$ and maintained at each $\Delta T^{\prime}$ for more than $2 \times 10^{5}$ $4 \times 10^{5}$ time steps in the present calculation. The iterative calculation up to the step length of applied temperature difference $\delta T^{\prime}=0.01^{\circ} \mathrm{C}$ is necessary for finding the accurate moment corresponding to the onset of a subharmonic bifurcation. The evolutionary processes for determining the maximum of stream func- tions, the vorticity and temperature at the point of the free surface of the half-floating zone are recorded and the Fourier spectrum is analyzed at each step. It is accurate for finding the start of the subharmonic bifurcation, such as the frequency-locking at $f / 2, f / 4, f / 8$, $f / 16$, where $f$ is basic frequency.

\section{NUMERICAL RESULTS}

The present computational results show that subharmonic bifurcations of $f / 2^{n}(n=2,3,4)$ successively appear as increasing applied temperature difference across the liquid bridge. The evolutionary processes of the temperature at a fixed point $y=0.16 \mathrm{~mm}$ on the free surface are given in Fig. $2 a-h$. The critical applied temperature difference for onset of oscillation is $\Delta T_{\mathrm{c}}^{\prime}=40^{\circ} \mathrm{C}$, at which the convection transfers from steady and symmetric one to oscillatory and asymmetric one, as shown in Fig. 2a. Figure $2 b$ and $c$ shows similar evolutionary temperature profiles to Fig. 2a for $40^{\circ} \mathrm{C} \leqslant \Delta T^{\prime} \leqslant 55.25^{\circ} \mathrm{C}$ or $1 \leqslant R \leqslant 1.38125$, where ratio $R$ is introduced as $R=\Delta T^{\prime} / \Delta T_{\mathrm{c}}^{\prime}$. The shape of the temperature profile is obviously different from the ones in Fig. $2 \mathrm{a}-\mathrm{c}$ if the $\Delta T^{\prime}$ increases slightly, such as $0.01^{\circ} \mathrm{C}$, in addition to $55.25^{\circ} \mathrm{C}(R=1.38125)$. The temperature records are still periodic at $\Delta T^{\prime}=58.9^{\circ} \mathrm{C}$ or $R=1.4725$ and $\Delta T^{\prime}=59.65^{\circ} \mathrm{C}$ or $R=1.49125$, and are followed by non-periodic pattern at $\Delta T^{\prime}=70^{\circ} \mathrm{C}$ or $R=1.75$, as shown in Fig. $2 \mathrm{~g}$. It is observed from Fig. $2 \mathrm{~h}$ that the temperature record becomes irregular and non-periodic, and the state at $\Delta T^{\prime}=100^{\circ} \mathrm{C}$ or $R=2.5$ seems to change to fully developed turbulent convection.

The temperature spectra are plotted in Fig. 3a-h, corresponding to the temperature profiles in Fig. 2. The computation of the spectra is accomplished by Fourier transforms. The patterns of temperature spectrum, as shown in Fig. $3 \mathrm{a}-\mathrm{c}$ for $R \leqslant 1.38125$, contain a sharp peak of the basic frequency $f$ and its harmonics at multiples of the basic frequency. The basic frequency increases slightly with increasing $R$. The first and second subharmonic bifurcations occur at the same time at $\Delta T^{\prime}=55.26^{\circ} \mathrm{C}$ or $R=1.3815$, and produce peaks at $f / 2, f / 3$ and $3 f / 4$, as shown in Fig. 3d. The $f / 8$ bifurcation appears at $\Delta T^{\prime}=58.9^{\circ} \mathrm{C}$ or $R=1.4725$, as shown in Fig. 3e, which is very weak at first, but grows with increasing $R$. The $f / 16$ bifurcations and corresponding subharmonics onset at $\Delta T^{\prime}=59.65^{\circ} \mathrm{C}$ or $R=1.49125$ with stronger background fluctuation spectra as given in Fig. $3 \mathrm{f}$. The spectra of frequency $f / 2^{n}(n>4)$ are mixed within the background fluctuation spectrum to further increase the applied temperature difference as shown in the Fig. $3 g$, and there are many close peaks, resulting in broadband background spectra. At $\Delta T^{\prime}=100^{\circ} \mathrm{C}$ or $R=2.5$ the spectrum becomes irregular and random, then the periodic convection of subharmonic bifurcation transfers to turbulent convection.

The process of transition from steady state to turbulence via subharmonic bifurcations is very complex, 

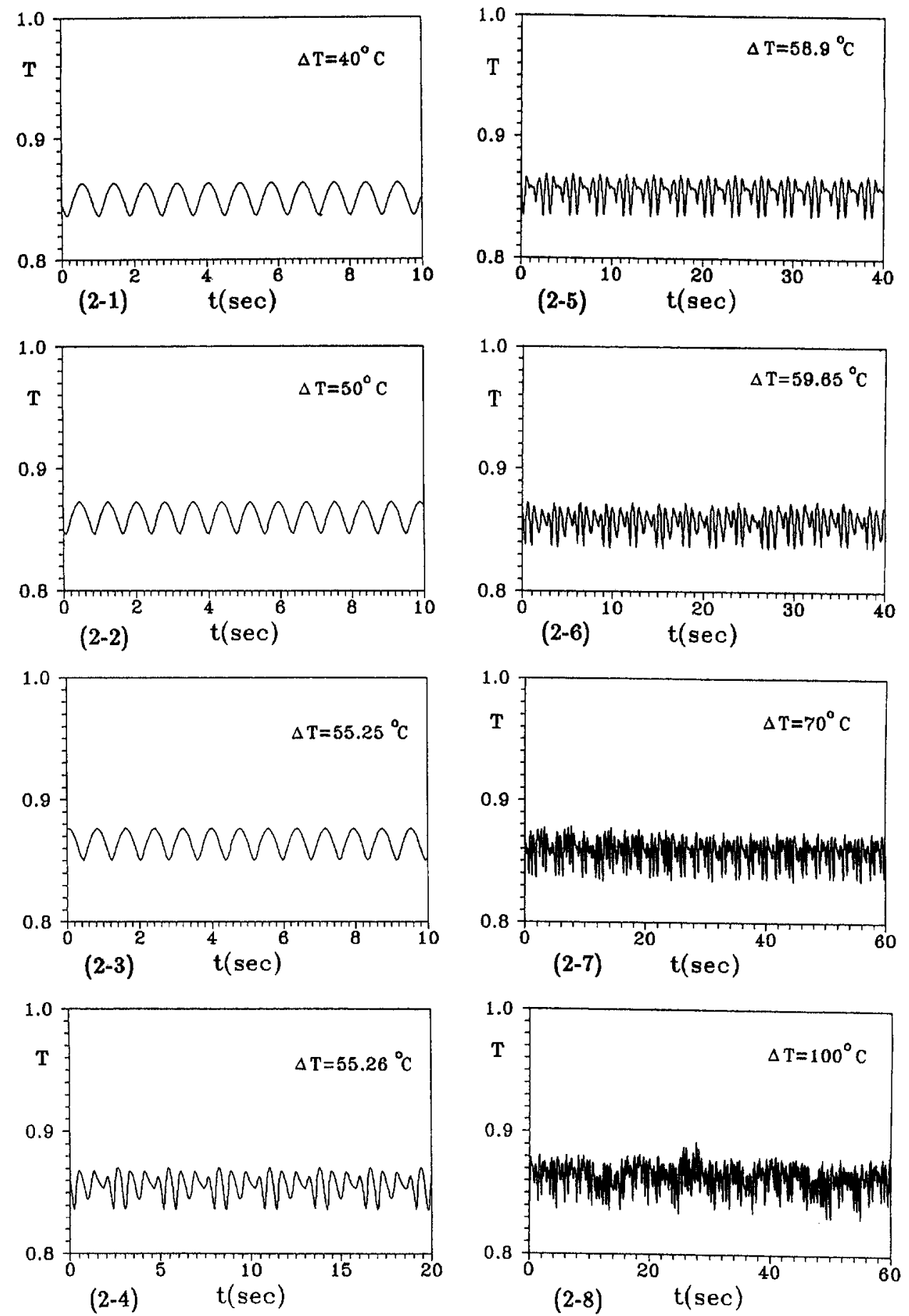

Fig. 2. The evolution of surface temperature at $y=0.16 \mathrm{~mm}$, and $D_{\text {min }} / D_{0}=0.9$.

the spectrum of $f / 8$ bifurcation appears for a short period at $\Delta T^{\prime} \geqslant 60^{\circ} \mathrm{C}$, and the $f / 18, f / 24$ bifurcations have been observed when the applied temperature difference is larger than $59.65^{\circ} \mathrm{C}$.

The temperature trajectories in the $(T, \dot{T})$ phase plane, corresponding to the evolutionary processes of temperature, are shown in Fig. $4 a-h$, where $\dot{T}$ is the differentiation of temperature with respect to time. There is only a single clockwise closed cycle in the phase plane plot in the range $40^{\circ} \mathrm{C} \leqslant \Delta T^{\prime} \leqslant 55.25^{\circ} \mathrm{C}$, as shown in Fig. 4a. Figure 4d shows that the bifurcation to $f / 4$ is revealed by four cycles with different trajectories, and the related temperature spectrum contains subharmonics with three frequencies $f, f / 2$, 


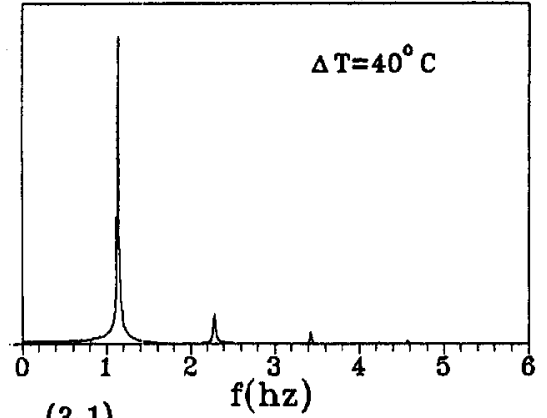

(3-1)

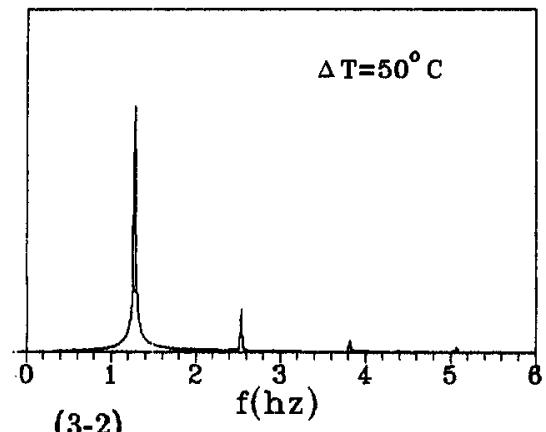

(3-2)

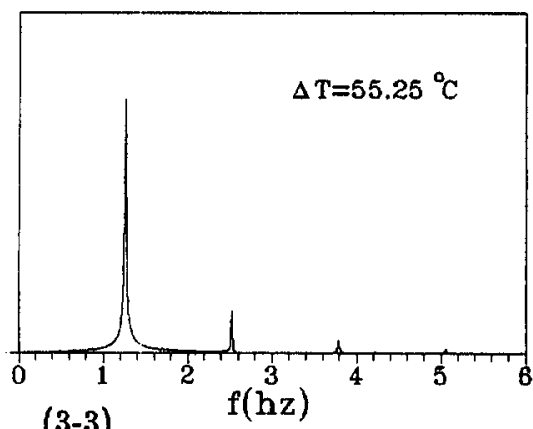

(3-3)

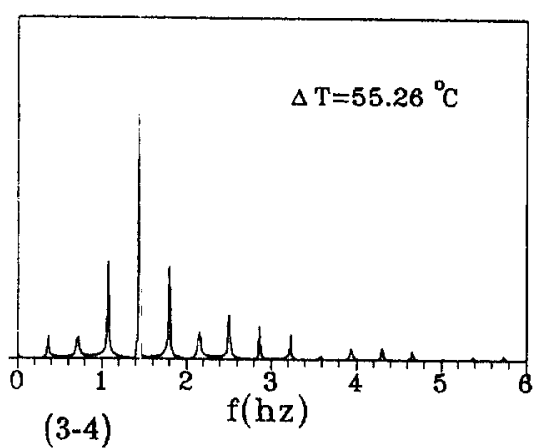

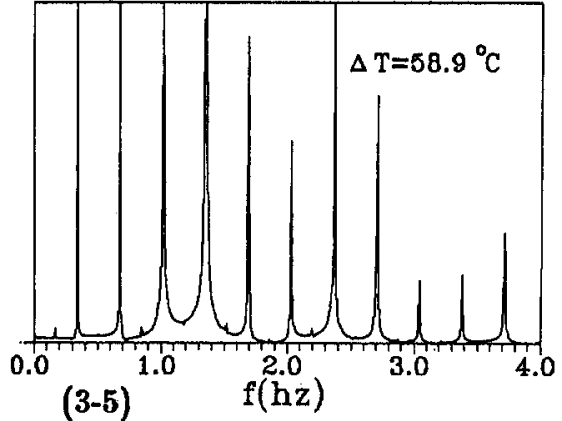
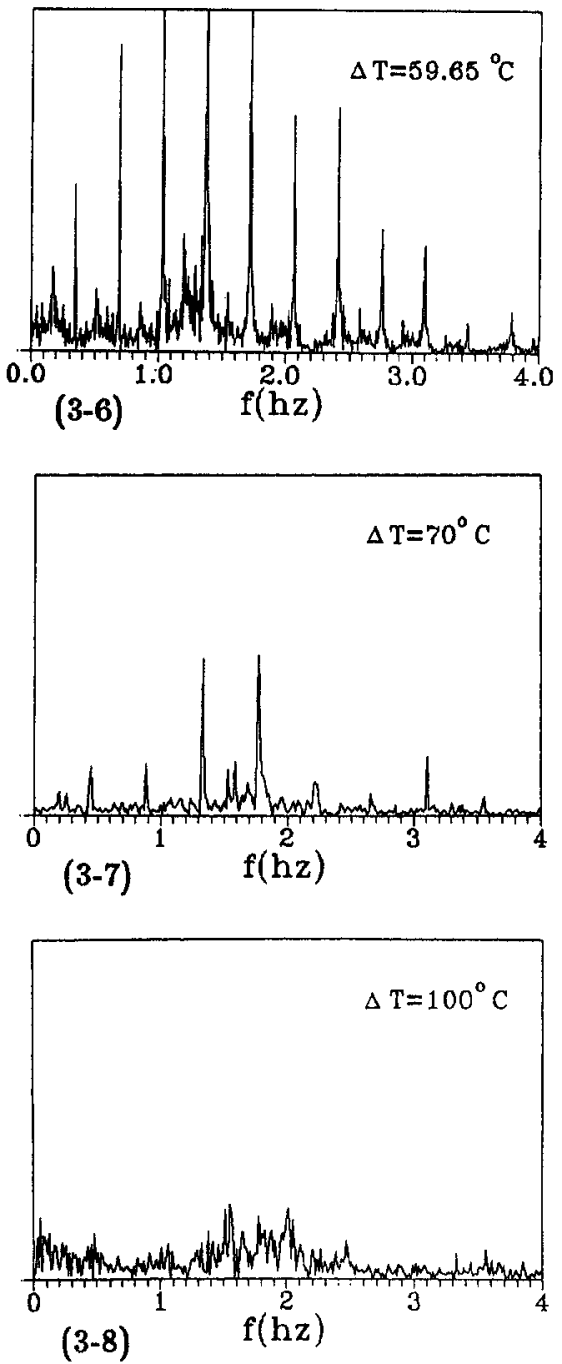

Fig. 3. The spectrum of surface temperature at $y=0.16 \mathrm{~mm}$, and $D_{\min } / D_{0}=0.9$.

and $f / 4$. The subsequent period-doubling bifurcation with increasing $\Delta T^{\prime}$ can be observed from Fig. $4 \mathrm{e}$ and $f$ by the gradual splitting of the trajectories in the phase plane plot. Many trajectories are wound together if $\Delta T^{\prime}$ is further increased, for example, $100^{\circ} \mathrm{C}$ as shown in Fig. 4h. The relevant Fourier spectrum in Fig. 3 h gives no sharp spectral line, and the convection becomes fully developed turbulence.
The time-dependent evolutionary processes of the asymmetric flow pattern and iso-thermal counters are given, respectively, in Figs. 5 and 6 in a period with interval sequence of $1 / 8$ period for applied temperature difference $\Delta T^{\prime}=40^{\circ} \mathrm{C}$. When $\Delta T^{\prime}$ increases up to $58.5^{\circ} \mathrm{C}$, the patterns of thermocapillary oscillatory convection with $f / 4$ bifurcation in a period of the basic frequency no longer exhibit periodicity, as 


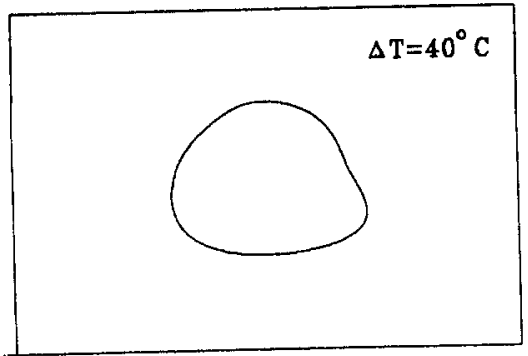

$(4-1)$

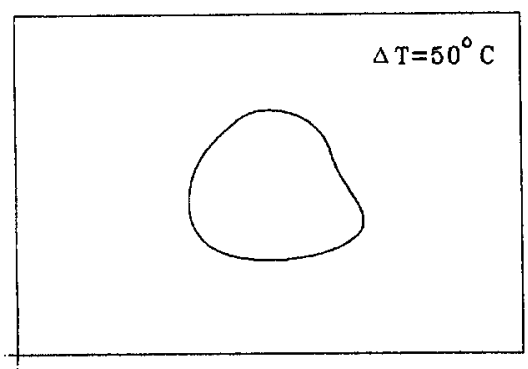

$(4-2)$

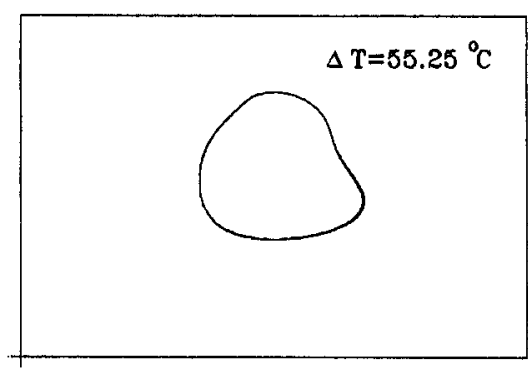

(4-3)

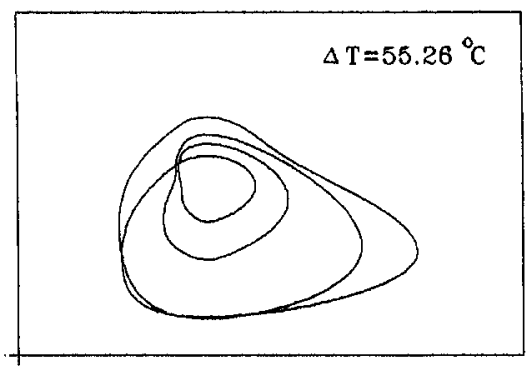

(4-4)

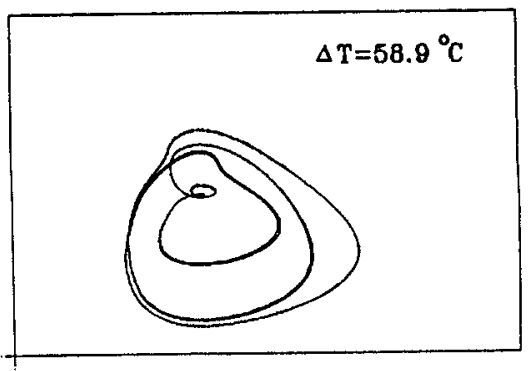

(4-5)

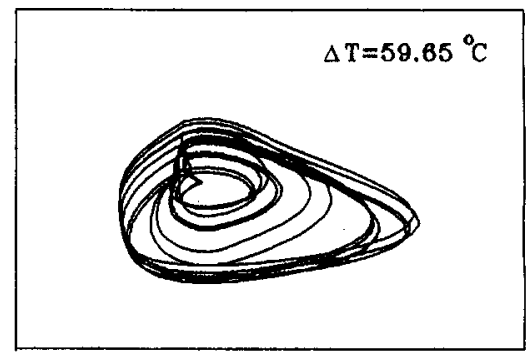

$(4-6)$

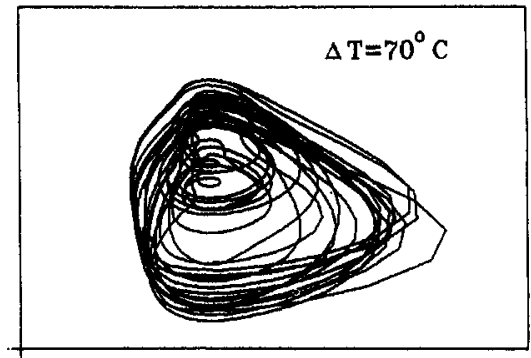

$(4-7)$

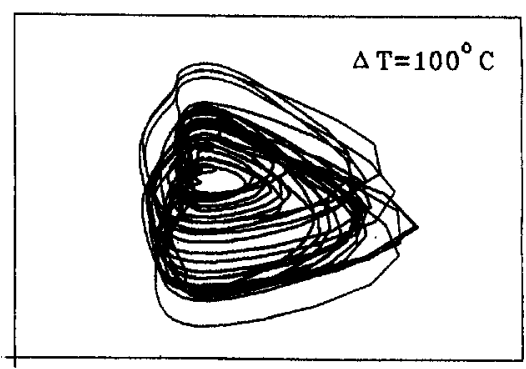

(4-8)

Fig. 4. The phase plane plots of surface temperature at $y=0.16 \mathrm{~mm}$ and $D_{\min } / D_{0}=0.9$.

shown in the patterns of Figs. 7 and 8 , and the multicells structure can be observed in the flow patterns. The maximum stream function and vorticity at the point of the free surface have similar Fourier spectra, with same frequencies but different amplitudes.

From Feigenbaum's general theory for the fluctuation spectrum of the route to turbulence, the value $a_{n}$ of the constraint should asymptotically approach the following relation:

$$
\delta_{n}=\frac{a_{n+1}-a_{n}}{a_{n+2}-a_{n+1}} \Rightarrow \delta,
$$

where $\delta=4.6642016$. According to present results, 


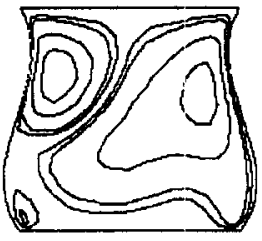

(a)

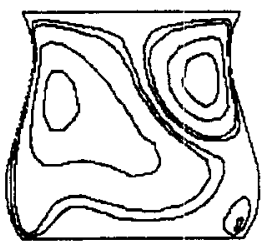

(e)

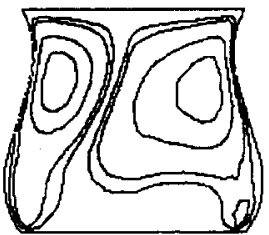

(b)

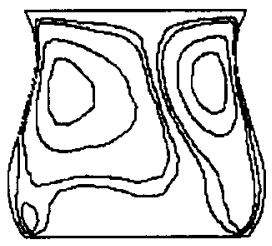

(f)

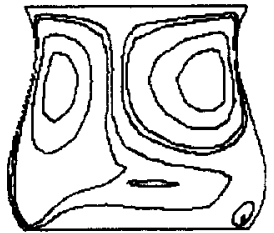

(c)

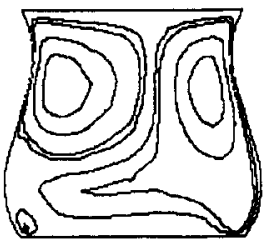

(g)

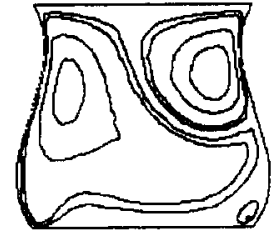

(d)

Fig. 5. The flow patterns in a period for $D_{\min } / D_{0}=0.9$ and $\Delta T^{\prime}=40^{\circ} \mathrm{C}$.

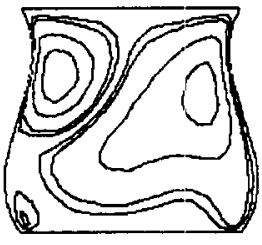

(a)

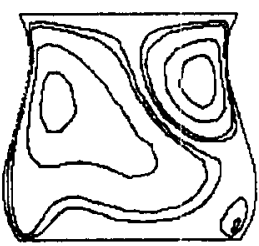

(e)

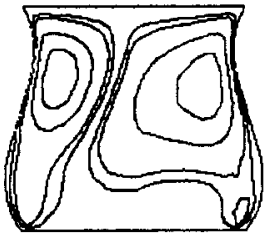

(b)

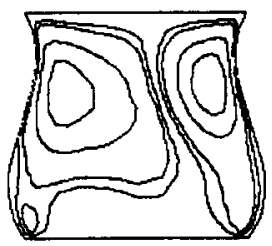

(f)

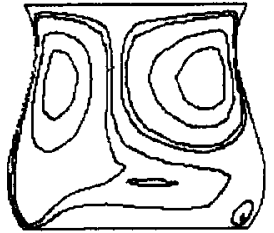

(c)

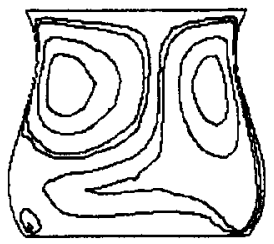

(g)

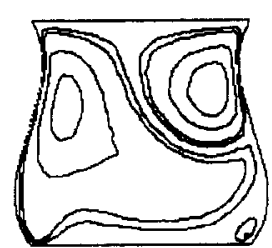

(d)

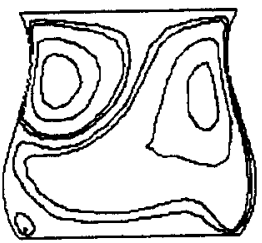

(h)

Fig. 6. The isothermal counters of the liquid bridge for $D_{\min } / D_{0}=0.9$ and $\Delta T^{\prime}=40^{\circ} \mathrm{C}$.

the Feigenbaun number computed for the bifurcations to the frequencies $f / 4, f / 8$ and $f / 16$ is

$$
\delta_{4}=\frac{f_{8}-f_{4}}{f_{16}-f_{3}}=\frac{58.9-55.26}{59.65-58.9}=4.853 .
$$

The result can be compared with theoretical values given by Feigenbaun. On the other hand, the average value of the odd multiples is computed from the Fourier spectrum, Fig. $4 \mathrm{f}, \mu_{4 / 8}=4.783$ and $\mu_{8 / 16}=1.837$. These values are lower than the theoretical values.

\section{CONCLUSION}

Thermocapillary convection driven by the non-uniformity of surface tension is a typical type of dissipative system, different from Benard convection driven by buoyancy. There are many differences between these two kinds of convection, and the transition features for both convections are attractive from the viewpoint of the dissipative system. Transition features of thermocapillary convection in the halffloating zone have been studied since the mid-1970s, and most interest concentrates on the onset of ocillation, which concerns only the start of the route to turbulence. There is a critical applied temperature difference or critical Rayleigh number to describe the onset of convection for Benard convection. However, the critical applied temperature difference or the critical Marangoni number is defined by the onset of oscillation for thermocapillary convection, because a small non-uniformity of surface tension due to applied temperature difference may induce convection. In the pre- 


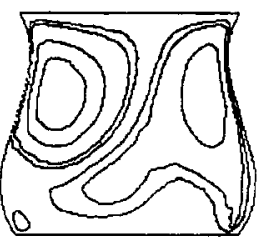

(a)

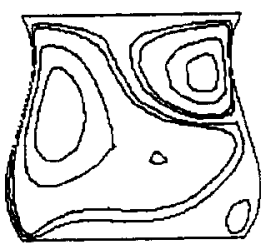

(e)

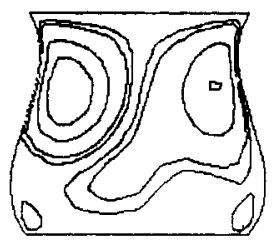

(b)

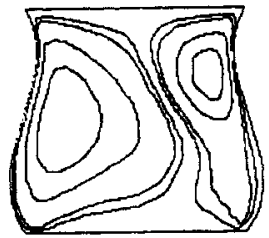

(f)

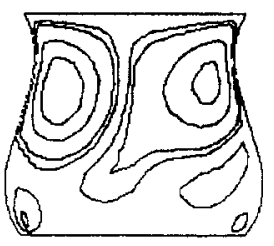

(c)

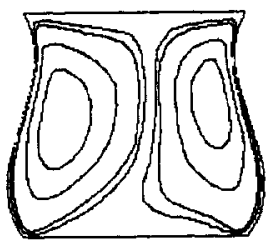

(g)

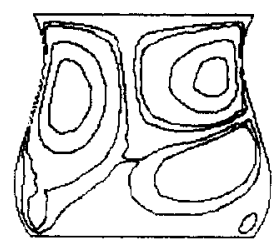

(d)

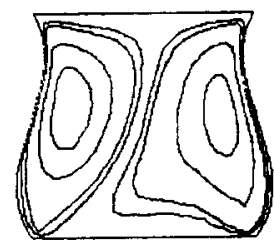

(h)

Fig. 7. The flow patterns in a period for $D_{\min } / D_{0}=0.9$ and $\Delta T^{\prime}=58.5^{\circ} \mathrm{C}$.

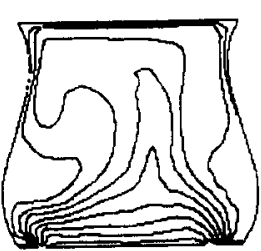

(a)

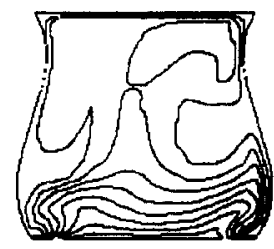

(e)

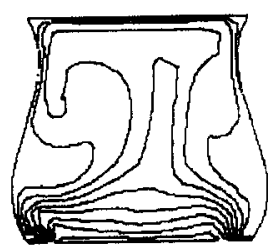

(b)

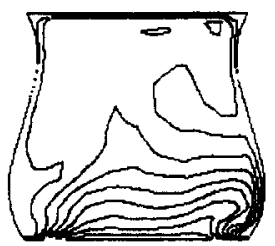

(f)

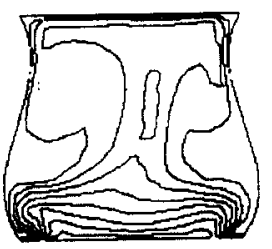

(c)

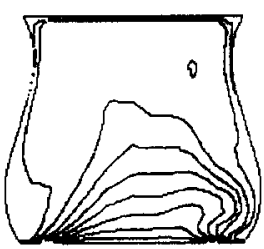

(g)

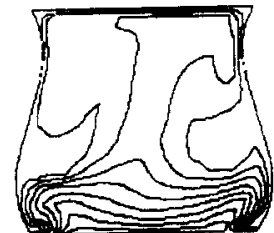

(d)

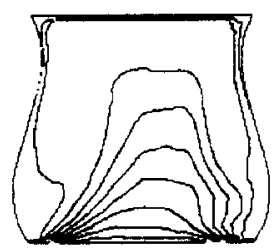

(h)

Fig. 8. The isothermal counters of the liquid bridge for $D_{\min } / D_{0}=0.9$ and $\Delta T^{\prime}=58.5^{\circ} \mathrm{C}$.

sent paper we present a complete route from steady convection to turbulence, but not limited to the route near the onset of oscillation of the thermocapillary convection.

There are many routes to turbulence, depending on the Prandtl number, Rayleigh number and geometric aspect for Benard convection, as shown in ref. [2], and a fixed route will be determined for fixed parameters. In the present paper, a typical geometric aspect of $A=1$ and liquid volume of $D_{\min } / D_{0}=0.9$ were adopted for the case of small Bond number and larger Prandtl number. The results show clearly the route from steady convection to turbulent convection via the period doubling bifurcations, and the Feigenbaum constant obtained by numerical test is close to the theoretical value. It means that the route of subharmonic bifurcations is typical in the transition process of thermocapillary convection. The results of sub- harmonic bifurcation support the idea that the oscillation in the liquid bridge of floating zone is induced by the internal instability. However, as that kind of instability may excite the right features, it should be studied carefully. Furthermore, it will be interesting to study and to find the transition process for other parameter ranges, and whether there are other routes to turbulence for thermocapillary convection.

The numerical simulation of an unsteady twodimensional model was investigated to show the transition process. It seems that the results discover the intrinsic physical features of chaos. It will be of benefit to do the numerical simulation of an unsteady threedimensional model for half-floating-zone convection. On the other hand, it is necessary to study experimentally the transition process of half-floating-zone convection, which also gave the bifurcation structure 
in the laboratory on the grounds of a liquid bridge with small typical scale, and will be discussed elsewhere. Experiments in the microgravity environment in the future will be useful.

\section{REFERENCES}

1. M. J. Feigenbaur, Phys. Lett. 74A, 375 (1979).

2. J. P. Gollub and S. V. Benson, J. Fluid Mech. 100, 449 (1980).

3. M. Giglio, S. Musazzi and U. Perini, Phys. Rev. Lett. 47, 243 (1981).

4. C. E. Chang and W. R. Wilcox, J. Crystal Growth 28,8 (1975).

5. C.-H., Chun, Acta Astronaut. 6, 1073 (1979).

6. F. Preisser, D. Schwabe and A. Scharmann, J. Fluid Mech. 126, 545 (1983).
7. W. R. Hu, H. T. You and Z. H. Cao, Sci. Sin. A35, 1001 (1992).

8. J. Z. Shu, Y. L. Yao and W. R. Hu, Sci. Sin. A36, 326 (1993).

9. M. K. Smith and S. H. Davis, J. Fluid Mech. 132, 119 (1983).

10. M. K. Smith and S. H. Davis, J. Fluid Mech. 132, 145 (1983).

11. H. C. Kuhlmann and H. J. Rath, J. Fluid Mech. 247, 247 (1993)

12. Z. M. Tang and W. R. Hu, Adv. Space Res. 13, 86 (1993).

13. W. R. Hu and Z. M. Tang, Int. J. Heat Mass Transfer 37, 1563 (1994).

14. S. Ostrach, Y. Komotani and C. L. Lai, PCH Physicochemical Hydrodynamics 6, 585 (1985).

15. Z. M. Tang and W. R. Hu, Micrograv. Q. 2, 123 (1992). 